\title{
Early Universe Plasma Separation and the Creation of a Dual Universe
}

\author{
Mohammed. B. Al-Fadhli ${ }^{*}$ \\ ${ }^{1}$ College of Science, University of Lincoln, Lincoln, LN6 7TS, UK. \\ *Correspondence: malfadhli@lincoln.ac.uk; mo.fadhli7@gmail.com
}

\begin{abstract}
The Planck Legacy recent release revealed a closed and positively curved early universe with a confidence level greater than $99 \%$. In this study, the Friedmann-LemaitreeRobertson-Walker (FLRW) metric is enhanced to model early universe plasma, incorporating its reference curvature radius upon the emission of the cosmic microwave background (CMB) and the reference scale factor of the energy flux. The universe evolution from early plasma is modelled utilising quantised spacetime worldlines, where they revealed both positive and negative solutions implying that matter and antimatter in the plasma could be separated by electromagnetic fields and evolved in opposite directions as distinct sides of the universe, corroborating the $\mathrm{CMB}$ dipole anisotropy. The model indicates a nascent hyperbolic expansion is followed by a first phase of decelerating expansion during the first $\sim 10 \mathrm{Gyr}$, and then, a second phase of accelerating expansion. The model theoretically resolves the tension in Hubble parameter measurements, with a predicted density at the phase transition of $\sim 1.16$. Further, it predicts a final time-reversal phase of rapid spatial contraction leading to a Big Crunch, signalling a cyclic universe. Simulations of the quantised spacetime continuum flux through its travel along the predicted worldlines demonstrated the fast-orbital speed of stars resulting from an external momentum exerted on galaxies via the spatial curvature through imaginary time dimension. These findings indicate that early universe plasma could be separated and evolved into distinct sides, collectively and geometrically influencing the universe evolution.
\end{abstract}

Keywords: Plasma, accelerated expansion, parallel universe, duality, antimatter.

\section{INTRODUCTION}

Advances in cosmology and astronomical observations over the last two decades have revealed many inconsistencies with the standard model of cosmology of a spatially flat universe, the lambda cold dark matter model $(\Lambda \mathrm{CDM})$. The recent Planck Legacy (PL18) release indicated the existence of an enhanced lensing amplitude in the CMB, which is higher than that estimated by $\Lambda \mathrm{CDM}$. This endorses a closed and positively curved early universe with a confidence level more than 99\% (Aghanim et al., 2020; Di Valentino, Melchiorri and Silk, 2020). Besides, the observed gravitational lensing by substructures of several galaxy clusters is an order of magnitude more than that estimated by $\Lambda \mathrm{CDM}$ (Meneghetti et al., 2020; Umetsu, 2020). Furthermore, the well-known 120 orders of magnitude discrepancy between the estimated vacuum energy density by $\Lambda \mathrm{CDM}$ for a flat accelerated expanding universe and the energy cutoff calculations of the vacuum energy density by the quantum field theory (QFT) (Rugh and Zinkernagel, 2000). Therefore, this evidence endorses a spatially curved universe in spite of the spacetime flatness of the local/present universe.
The early universe was composed of hot and opaque plasma at thermal equilibrium and highest density (Weinberg, 1972; Holcomb and Tajima, 1989), whereas the expansion of the closed early universe reduced universe's density and increased its entropy. In contrast with the $\Lambda \mathrm{CDM}$ assumption of antimatter elimination, the matter and antimatter in the plasma could be separated by electromagnetic fields (Klein, 1966, 1971) due to the plasma drift phenomenon, consequently, evolving in opposite directions. This scenario corresponds the CMB dipole anisotropy and the quantum electrodynamics theory (QET), where advanced measurements of the fine structure of hydrogen and antihydrogen atoms were found to be consistent with predictions of QET with a precision of $2 \%$, including the Lamb shift feature (Ahmadi et al., 2020). These measurements corroborate the concept of identical matter and antimatter apart of their opposite charge and spin, which can undermine the elimination assumption. Sings of plasma separation or a significant predominant event might be observed as the associated noise surrounding the measured gravitational waves (Creswell et al., 2017). 
In 2020, Riess found that the expansion of the universe is faster than $\Lambda \mathrm{CDM}$ estimations with the disagreement between measurements taken from the early universe based on the $\mathrm{CMB}$ and the present universe using the type Ia supernovae distanceredshift method is four to six standard deviations (Riess, 2020), while Ryskin demonstrated that the vacuum energy cannot be inducing this accelerated expansion (Ryskin, 2020). Further, $\Lambda C D M$ assumes a homogeneous and isotropic universe on large scales. Although this assumption could be consistence with the early universe, the observed voids and galaxy filaments indicate an anisotropic universe (Albert Robson, 2019). Besides, the universe expanding rate is observed to vary depending on the direction while precise measurements of the fine structure constant over multiple directions in several studies revealed it varies with time and across a specific axis (Uzan, 2011; Wilczynska et al., 2020). This indicates a strong anisotropy at a five-sigma confidence level (Migkas et al., 2020). Therefore, emerging evidence supports a growing need for profound adjustments to $\Lambda \mathrm{CDM}$ or new physics (Lusso et al., 2019).

In this study, an alternative cosmological model of a closed early universe is considered. The universe evolution from the early universe plasma is modelled utilising quantised spacetime continuum worldlines. The paper is organised as follows. Section 2 presents the plasma modelling, its boundary contribution and the derivation of the quantised worldlines. Sections 3 discusses the universe evolution and its expansion rate while Section 4 simulates the curvature of the universe and the spiral galaxy orbital speed. Section 5 summarises the outcomes and conclusions. Finally, Section 6 suggests future works.

\section{Mathematical Model}

In addition to the PL18 evidence of a positively curved early universe, the closed finite universe can provide an agreement with the $\mathrm{CMB}$ anisotropy observations (Efstathiou, 2003) and could explain the quantum entanglement (QE), where the energy of the finite universe is conserved, thus cosmic conservation governs the total spin of a pair of particles to be conserved regardless of their locations. Otherwise, unpreserved total spin breaks energy conservation of the finite closed universe (Al-Fadhli, 2020b).

The measured gradual variation in the finestructure 'constant' (Donoghue, 2003; Uzan, 2011; Wilczynska et al., 2020) can be a result of the evolution of the universe's density due to its expansion, which affects the travel speed of light. These measurements may reveal that the presumed fundamental constants might be dependent on other universe properties.
According to Mach's principle, Schrödinger in 1925 pointed to the reliance of the Newtonian gravitational 'constant' $G$ on the distribution of universe's masses and the universe's radius of curvature $r$ while Dirac in 1938 proposed its correlation to the universe's age (Dirac, 1938), i.e., $G_{\eta}$ and $r_{\eta}$ could be functions of the conformal time $\eta$.

Alternatively, this study sought a fundamental constant relying on the energy conservation law, as it is a firm fundamental law within a closed system (closed universe). Because of the spacetime curving nature according to general relativity (GR), a new constant is defined as spacetime continuum modulus of deformation/curvature $E_{D}$. Spacetime is regarded as a continuum having a dual quantum nature that it curves as waves and, travels as quantum particles at the speed of light; where the latter is justified due to the energy flux from early universe plasma into space at the speed of light creating 'spacetime continuum', 'quintessence', 'vacuum energy', or 'darknesses'. Recent findings regarding light polarization from the $\mathrm{CMB}$ support the existence of an exotic substance through space causing these measured polarizations (Minami and Komatsu, 2020). This corroborates the concept of spacetime continuum quantum duality. By using Einstein field equations, $E_{D}=($ stress/strain) in $\left(\mathrm{N} / \mathrm{m}^{2}\right)$ is expressed as

$E_{D}=\frac{T_{v}^{\mu}-T \delta_{v}^{\mu} / 2}{R_{v}^{\mu} / \mathcal{R}}=\frac{\mathrm{c}^{4}}{8 \pi G_{\eta} r_{\eta}^{2}}$

where the stress is signified by the stress-energy tensor $T_{v}^{\mu}$ of trace $T$, while the strain is signified by the Ricci curvature tensor $R_{v}^{\mu}$ as the change in curvature divided by $\mathcal{R}=1 / r_{\eta}^{2}$, the scalar of the preexisting curvature, and $r_{\eta}$ is the universe curvature radius. According to the theory of elasticity, the modulus times the volume equals the internal energy of reversible systems (Landau, 1986). Thus, $E_{D}$ could represent the internal energy density of the space (vacuum energy density). $E_{D}$ is proportional to the fourth-order of $c$, resembling the QFT energy cut-off prediction of the vacuum energy density (Rugh and Zinkernagel, 2000). Further, because the modulus $E_{D}$ is a constant (Landau, 1986), Eq. (1) confirms the reliance of $G_{\eta}$ on the universe curvature radius $r_{\eta}$.

By incorporating the background/pre-existing universal curvature as function of the conformal time represented by the scalar curvature $\mathcal{R}$ while $E_{D}$ is utilised to maintain the compatibility of the extended Einstein-Hilbert action as

$S=\int\left[\frac{E_{D}}{2} \frac{R}{\mathcal{R}}+\mathcal{L}_{M}\right] \sqrt{-g} d^{4} x$

where $R$ is the Ricci scalar curvature, $\mathcal{L}_{M}$ is the Lagrangian density and $g$ is the determinant of the metric tensor $g_{u v}$. 
The derivations of the extended action are given in (Al-Fadhli, 2020a) as

$R_{\mu \nu}-\frac{1}{2} R \hat{g}_{\mu \nu}+\frac{R-\mathcal{R}}{\mathcal{R}}\left(K_{\mu \nu}-\frac{1}{2} K \hat{q}_{\mu \nu}\right)=\frac{8 \pi G_{\eta}}{c^{4}} T_{\mu \nu}$

The extended field equations in Eqs. (3) consist of the conformally transformed Einstein field equations and the boundary term, in addition to the energy-stress term on the right-hand side; where $\hat{g}_{\mu \nu}=g_{\mu \nu}+2 \bar{g}_{\mu \nu}=$ $g_{\mu \nu}+2 \mathcal{R}_{\mu \nu} / \mathcal{R}$ is the conformal transformation of the metric tensor due to the fact that Einstein spaces are a subclass of the conformal space (Kozameh et al., 1985). $K_{\mu v}$ is the extrinsic curvature tensor and $\hat{q}_{u v}$ denotes the conformally transformed induced metric tensor on the spacetime boundary manifold. The conformal transformation can describe the tidal distortion of gravitational waves in the absence of matter (Penrose, 2005) and the galaxy rotation curve as it accounts for the universe curvature evolution over the universe's age. The boundary tensor/term is only significant at the high-energy limit such as in black holes (Dyer and Hinterbichler, 2009) and the early universe, and can remove the singularities from the theory.

\subsection{Early Universe Plasma Model}

The FLRW metric is the standard cosmological metric model, which assumes an isotropic and homogenous universe (Ellis and van Elst, 1998; Lachì Eze-Rey and Luminet, 2003), where the isotropy and homogeneity of the early universe plasma based on the CMB are consistent with this metric model. The PL18 release revealed a closed and positively curved early universe. Therefore, the plasma reference radius of curvature $r_{P}$ upon the emission of the $\mathrm{CMB}$ and the corresponding early universe scale factor $a_{P}$ of the energy flux as shown in Figure 1 at the reference time $t_{p}$ are incorporated to enhance the metric model. Thus, the four-dimensional spacetime interval $d s$ of the enhanced metric is

$$
\begin{aligned}
d s^{2}=c^{2} d t^{2}-\frac{a^{2}}{a_{p}^{2}}( & \frac{d r^{2}}{1-\left(r^{2} / r_{p}^{2}\right)}+r^{2} d \theta^{2} \\
& \left.+r^{2} \sin ^{2} \theta d \phi^{2}\right)
\end{aligned}
$$

where $a / a_{p}$ is the new dimensionless scale factor and $r, \phi, \theta$ are the comoving coordinates. Accordingly, the metric components are

$g_{t t}=c^{2}, \quad g_{r r}=-\left(\frac{a^{2}}{a_{p}^{2}}\right) /\left(1-\frac{r^{2}}{r_{p}^{2}}\right)$,

$g_{\theta \theta}=-\left(\frac{a^{2}}{a_{p}^{2}}\right) r^{2}, \quad g_{\phi \phi}=-\left(\frac{a^{2}}{a_{p}^{2}}\right) r^{2} \sin ^{2} \theta$.

No conformal distortion is included in this metric, so its outcomes are comparable with literature.
Figure 1 shows the enhanced metric model of the of early universe plasma expansion

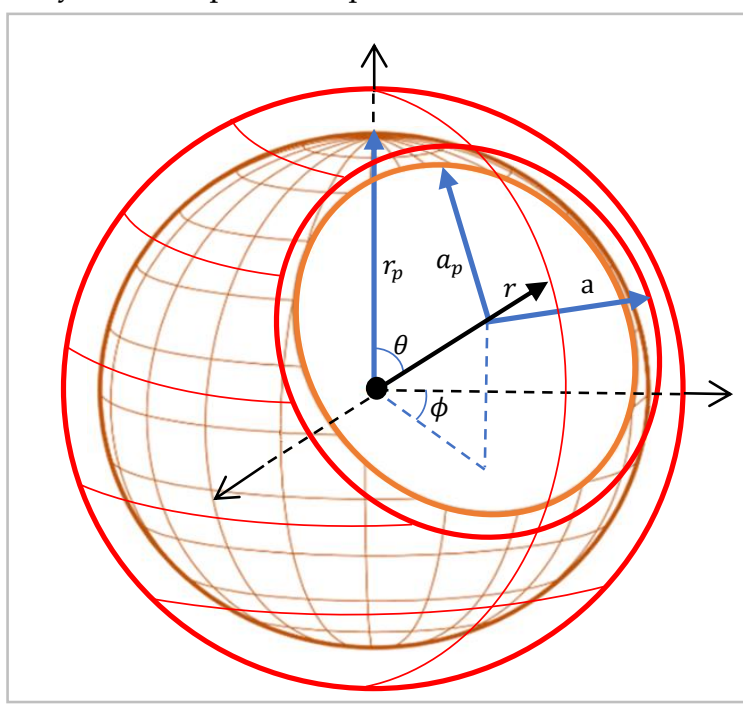

Figure 1. The hypersphere of a positively curved early universe plasma expansion upon the $\mathrm{CMB}$ emissions. $r_{p}$ is the reference radius of the intrinsic curvature and $a_{p}$ is the reference scale factor of the early universe energy flux at the corresponding reference time $t_{p} \cdot a / a_{P}$ denotes the new dimensionless scale factor of this metric model. $r, \phi, \theta$ are the comoving coordinates.

The Ricci curvature tensor $R_{u v}$ is solved using Christoffel symbols for $g_{u v}$ in Eqs. (5) as well as the Ricci scalar curvature $R$ (the derivation in Appendix A) as follows

$R_{r r}=\frac{1}{c^{2}}\left(\frac{a \ddot{a}}{a_{p}^{2}}+\frac{2 \dot{a}^{2}}{a_{p}^{2}}+\frac{2 c^{2}}{r_{p}^{2}}\right) /\left(1-\frac{r^{2}}{r_{p}^{2}}\right)$,

$R_{\theta \theta}=\frac{r^{2}}{c^{2}}\left(\frac{a \ddot{a}}{a_{p}^{2}}+\frac{2 \dot{a}^{2}}{a_{p}^{2}}+\frac{2 c^{2}}{r_{p}^{2}}\right)$,

$R_{\phi \phi}=\frac{r^{2} \sin ^{2} \theta}{c^{2}}\left(\frac{a \ddot{a}}{a_{p}^{2}}+\frac{2 \dot{a}^{2}}{a_{p}^{2}}+\frac{2 c^{2}}{r_{p}^{2}}\right)$,

$R_{t t}=-3 \frac{\ddot{a}}{a}, R=\frac{-6}{c^{2}}\left(\frac{\ddot{a}}{a}+\frac{\dot{a}^{2}}{a^{2}}+\frac{c^{2} a_{p}^{2}}{a^{2} r_{p}^{2}}\right)$.

By solving Einstein field equations $R_{\mu \nu}-\frac{1}{2} R g_{\mu \nu}=$ $\frac{8 \pi G_{\eta}}{\mathrm{c}^{4}} T_{\mu \nu}$ for a perfect fluid given by $T_{\mu \nu}=(\rho+$ $\left.\frac{P}{c^{2}}\right) u_{\mu} u_{v}+P g_{\mu v}$ (Straumann, 2013) then substituting Eqs. $(5,6)$, the Friedmann equations that count for the plasma reference curvature radius and the reference scale factor are

$H^{2} \equiv \frac{\dot{a}^{2}}{a^{2}}=\frac{8 \pi G_{\eta} \rho}{3}-\frac{c^{2} a_{p}^{2}}{a^{2} r_{p}^{2}}$,

$\dot{H} \equiv \frac{\ddot{a}}{a}=-\frac{4 \pi G_{\eta}}{3}\left(\rho+3 \frac{P}{c^{2}}\right)$.

where $H, P$, and $\rho$ are the Hubble parameter, pressure, and density respectively. The equations do not show a conformal distortion as the conformal transformation is not applied in the current study. 


\subsection{Universe Evolution Model}

The Friedman equations in Eqs. $(7,8)$ are solved over the imaginary conformal time of one universe life cycle by rewriting Eq. (7) in terms of the imaginary conformal time in its parametric form $d \eta=-i \frac{a_{p}}{a} d \tau$ while initiating at the reference imaginary time $\tau_{p}$ as

$\int_{0}^{\eta} d \eta=\int_{0}^{2 \pi} a_{p}\left(\frac{8 \pi G_{p} \rho_{p} a_{p}^{3}}{3} a-\frac{c^{2} a_{p}^{2}}{r_{p}^{2}} a^{2}\right)^{-1 / 2} d a$

where $\rho=\frac{\rho_{p} a_{p}{ }^{3}}{a^{3}}$ (Ryden, 2006) and $a$ is a function of the imaginary time $\tau=i$. By integrating, the scale factor evolution is

$a(\eta) / a_{p}=\frac{M_{p} G_{p}}{c^{2} r_{p}}\left(1-\cos \frac{c}{r_{p}} \eta\right)$

where $M_{p}=\frac{4}{3} \pi \rho_{p} r_{p}{ }^{3}$ is the plasma mass and $G_{p}$ is the gravitational parameter value at $\tau_{p}$. The constant in Eq. (10) can be written more fundamentally in terms of the modulus $E_{D}$ representing the vacuum energy density and the universe energy density $E$ using Eq. (1) as $E / 6 E_{D}$.

Additionally, the evolution of the imaginary time $\tau(\eta)$ can be obtained by integrating the length of the spatial factor contour of one universe life cycle over the expansion speed $H_{\eta}$ while initiating at the reference imaginary time $\tau_{p}$ with the corresponding spatial factor $a_{p}$. Thus, by rewriting Eq. (10) in terms of the Hubble parameter by its definition at $\tau_{p}$ as $d \tau=i \frac{d a}{H a_{p}}$ as

$\int_{\tau_{P}}^{\tau} d \tau=i \int_{0}^{\eta} \frac{E}{6 H_{\eta} E_{D}}\left(1-\cos \frac{c}{r_{p}} \eta\right) d \eta$

By performing the integration, the imaginary time evolution is

$\tau(\eta)=i \frac{E}{6 H_{\eta} E_{D}}\left(\eta-\sin \frac{c}{r_{0}} \eta\right)+\tau_{p}$

where $\tau_{p}$ denotes the reference imaginary time.

According to the energy conservation law, the stress-energy tensor divergence vanishes, $\Delta_{v} T^{u v}$, this yields $\frac{\dot{a}}{\mathrm{a}} T_{u}^{u}+3 \frac{\dot{a}}{\mathrm{a}} \rho-i \frac{\partial \rho}{\partial \tau}=0, \quad 3\left(\rho+\frac{P}{c^{2}}\right) \frac{\dot{a}}{\mathrm{a}}-i \frac{\partial \rho}{\partial \tau}=0$. By combining these outcomes, integrating, and then substituting the spatial scale factor rate in Eq. (10) to their outcome, the universe density evolution over the conformal time is

$\rho_{\eta}=D_{p}\left(1-\cos \frac{c}{r_{p}} \eta\right)^{-3}$

where $D_{P}$ is a constant. According to Eq. (8), the Hubble parameter is dependent on the density; thus, by substituting Eq. (13) to Eq. (8) and initiating the integration at $\tau_{p}$ thus, $\dot{H}=\frac{\ddot{a}}{a_{p}}$ as

$\int_{H_{p}}^{H} \dot{H}=\int_{0}^{\eta} \frac{-4 \pi G_{p} D_{p}}{3 a_{p}}\left(1-\cos \frac{c}{r_{p}} \eta\right)^{-3} d \eta$
By integration, the Hubble parameter evolution is

$H_{\eta}=H_{a}\left(\frac{1}{5} \cot ^{5} \frac{c}{2 r_{p}} \eta+\frac{2}{3} \cot ^{3} \frac{c}{2 r_{p}} \eta+\cot \frac{c}{2 r_{p}} \eta\right)+H_{p}$

where, $H_{a}=\frac{\pi G_{p} D_{p}}{3 a_{p}}$ and $H_{p}$ is the integration constant. Combining Eqs. $(10,12-15)$ in the complex frame results in the quantised hyper-spherical spacetime continuum wave function with respect the reference radius of curvature as the third quantisation:

$$
\begin{aligned}
\underset{0<\eta \leq 2 \pi}{\overrightarrow{\psi_{L}}(\eta) / r_{p}}=\mp \frac{E}{6 E_{D}}\left(\left(1-\cos \frac{c}{r_{p}} \eta\right)^{2}\right. \\
\left.+\frac{c^{2}}{H_{\eta}^{2} a_{p}^{2}}\left(\eta-\sin \frac{c}{r_{p}} \eta\right)^{2}\right)^{1 / 2} e^{i \cot \frac{H_{\eta} a_{p}\left(1-\cos \frac{c}{r_{p}} \eta\right)}{c\left(\eta-\sin \frac{c}{r_{p}} \eta\right)}}
\end{aligned}
$$

The third quantisation denotes the wave function $\overrightarrow{\psi_{L}}(\eta)$ of the spacetime continuum evolution that simultaneously propagates in space and oscillates in time where $E / E_{D}$ denotes a new dimensionless energy parameter as the ratio of the universe energy density to vacuum energy density. Additionally, under Weyl's quantum phase invariance (gauge invariance) $\psi(\eta)=\psi(\eta) e^{i \pi(\eta)}($ Straub, 2006).

\subsection{Plasma Boundary Contribution}

The positive and negative solutions of the quantised spacetime continuum wave function in Eq. (16) imply that matter and antimatter in the plasma are evolving in opposite directions. The plasma drift phenomenon is caused by the presence of the electromagnetic fields (Klein, 1966, 1971). In early plasma case consisting of matter and antimatter, the drift phenomenon drives matter and antimatter in opposite directions as they have an opposite electrical charge (Figure 2).

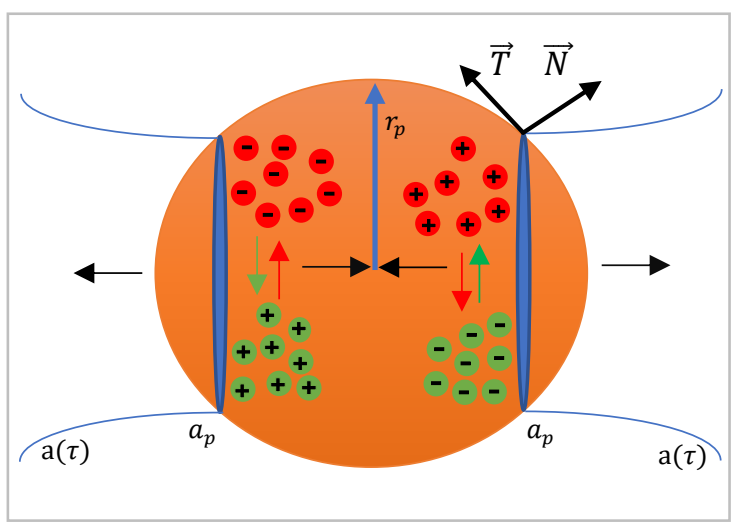

Figure 2. The evolution of matter and antimatter plasma. $\vec{N}$ and $\vec{T}$ are the normal and tangential vectors on the plasma surface respectively regarding the extrinsic curvature. $r_{p}$ is the reference radius of the intrinsic curvature and $a_{p}$ is the reference scale factor of the early universe energy flux at the corresponding reference imaginary time $\tau_{p}$. 
For high energy limits, the gravitational contribution of the plasma boundary can be obtained using the boundary term in the extended field equations in Eqs. (3), where at the reference imaginary time $\tau_{p}$, there is no conformal transformation. Therefore, the induced metric on the plasma hypersphere boundary $q_{\mu \nu}$ is given in Eqs. (17) (Pavel Grinfeld, 2013).

The extrinsic curvature tensor is solved utilising the formula $K_{u v}=-\overrightarrow{T_{\mu}} \cdot \nabla_{v} \overrightarrow{N_{v}}$. Due to the symmetry of the plasma hypersphere, the covariant derivative reduces to the partial derivative as follows $K_{u v}=$ $-\overrightarrow{T_{\mu}} \partial \vec{N} / \partial \overrightarrow{T^{v}}$ (Pavel Grinfeld, 2013):

$\begin{aligned} q_{\mu \nu} & =\left(\begin{array}{ccc}\mathrm{c}^{2} & 0 & 0 \\ 0 & \frac{a^{2}}{a_{P}{ }^{2}} R^{2} & 0 \\ 0 & 0 & \frac{a^{2}}{a_{P}{ }^{2}} R^{2} \sin ^{2} \theta\end{array}\right), \\ K_{\mu \nu} & =\left(\begin{array}{ccc}0 & 0 & 0 \\ 0 & -\frac{a^{2}}{a_{P}{ }^{2}} R & 0 \\ 0 & 0 & -\frac{a^{2}}{a_{P}{ }^{2}} R \sin ^{2} \theta\end{array}\right) .\end{aligned}$

The trace of the extrinsic curvature is $K=K_{\mu \nu} q^{\mu \nu}=$ $-2 / R$. The Gaussian curvature (intrinsic curvature) equals one over the square the radius of curvature. Thus, the pre-existing scalar curvature of the plasma boundary at $\tau_{p}$ is $\mathcal{R}_{p}=1 / r_{p}^{2}$.

On the other hand, the Ricci scalar curvature $R_{p}$ at $\tau_{p}$ can be written as the difference between kinetic and potential energy densities whereby substituting Friedmann equations in Eqs. $(7,8)$ into the Ricci scalar curvature in Eqs. (6) as

$R_{p}=\frac{6 G_{p}}{c^{2}}\left(\frac{4 \pi P_{p}}{c^{2}}-\frac{4 \pi \rho_{p}}{3}\right)$

By solving the boundary term $\frac{\mathcal{R}-R}{\mathcal{R}}\left(K_{\mu \nu}-\frac{1}{2} K q_{\mu \nu}\right)=$ $\frac{8 \pi G_{p}}{c^{4}} T_{\mu \nu}$ for a perfect fluid of $T_{\mu \nu}=\left(\rho+\frac{P}{c^{2}}\right) u_{\mu} u_{\nu}+P g_{\mu \nu}$ (Straumann, 2013) and choosing the normal $\vec{N}$ on the plasma boundary as a spacelike identity, and then substituting Eqs. $(18,17)$ into the boundary term as

$\frac{\frac{1}{r_{p}^{2}}-\frac{6 G_{p}}{c^{2}}\left(\frac{4 \pi P_{p}}{c^{2}}-\frac{4 \pi \rho_{p}}{3}\right)}{1 / r_{p}^{2}}\left(\frac{\mathrm{c}^{2}}{r_{p}^{2}}\right)=8 \pi G_{p} \rho_{p}$

By multiplying both sides by the plasma volume $V_{p}$ and simplifying, the reference radius of curvature $r_{p}$ is then

$r_{p}=\frac{4 G_{p} P_{p} V_{p}}{c^{4}}$

The reference curvature radius $r_{p}>0$ because any reduction in the plasma volume causes an increase in its pressure, whereas it is the smallest possible radius of the universe.

\section{Universe Evolution}

\subsection{Spacetime Continuum Worldlines}

A chosen mean evolution value of the Hubble parameter of $\sim 70 \mathrm{~km} \cdot \mathrm{s}^{-1} \cdot \mathrm{Mpc}^{-1}$ and a phase transition of expansion at universe age of $\sim 10 \mathrm{Gyr}$ are utilised to tune the integration constants of the derived model in Eq. (16) where the predicted energy density at the phase transition is $\sim 1.16$. The cosmic evolution of radiation coupled with matter/antimatter according to the quantised wave function model in Eq. (16) is predicted to experience three distinct phases, where due to symmetry, only the positive solution of one universe side is shown in Figure 3.

Firstly, the matter and antimatter sides of the universe expand in opposite directions away from the early universe plasma where they would be blue and red-shifted, corresponding the $\mathrm{CMB}$ dipole anisotropy. During the first phase (i.e., the first $\sim 10$ Gyr), the gradients of the worldlines indicate the expansion is decelerating. The expansion rate is discussed in detail in the next section. Though, in the second phase, the worldlines reverse their directions, with both sides entering a state of free fall towards each other. It is conceivable that both sides of matter and antimatter are free-falling towards each other at gravitational acceleration causing current accelerated expansion. The gradients of worldlines at this phase show an accelerated rate.

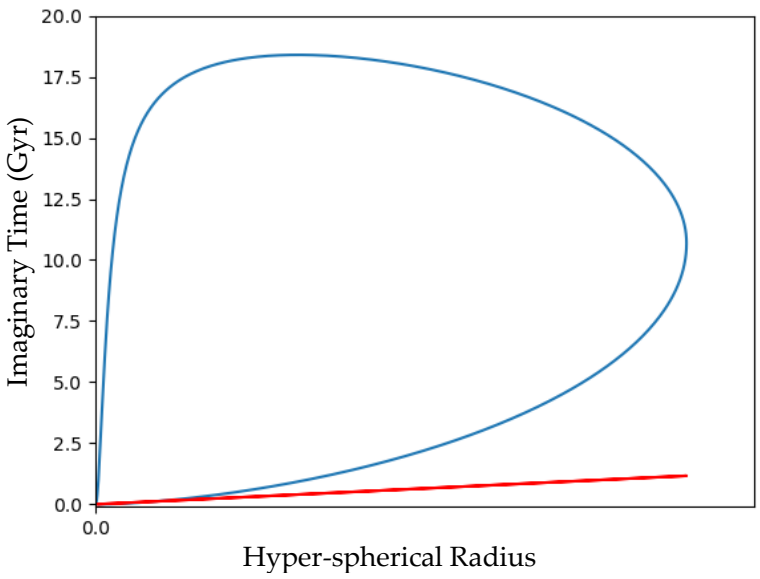

Figure 3. Evolution of the quantised wave function of the cosmic evolution of one side of the universe through space and time (blue curve) in addition to the straight line of light worldlines (diagram is not to scale). The evolutions are, at first, a phase of spatial expansion of both sides away from each other. Then, a second spatial expansion phase occurs in the reverse direction as both sides free-fall towards each other at gravitational acceleration. Finally, a third timereversal phase of spatial contraction. 
Interestingly, the model predicts a third phase of spatial contraction with a reversal-time arrow that appears after $\sim 18$ Gyr. In this phase, the universe experiences a contraction, which could be due to a high concentration of matter/antimatter at both sides, leading to the Big Crunch.

The free fall of both sides towards each other at gravitational acceleration can provide a physical explanation of current accelerated expansion while vacuum energy was found to be not accountable for this acceleration (Ryskin, 2020). Further, at the second phase, both sides are getting closer towards each other, which explains the reason behind the current increase in the average temperature of the universe (Chiang et al., 2020) in contrast to the state of cooling down from hot plasma during the first phase.

\subsection{Evolution of the Hubble Parameter}

The Hubble parameter, or the speed of spatial expansion, $H$, and the acceleration $\dot{H}$ can be determined using Eqs. (15) and (13 in 8), respectively. The predicted speed and acceleration of the spatial expansion are shown in Figure 4.

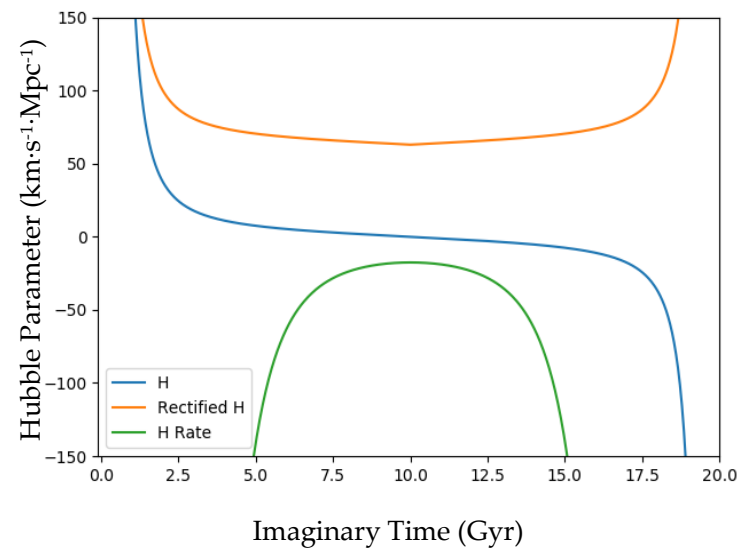

Figure 4. Hubble parameter (the speed of spatial expansion) and its acceleration.

The Hubble parameter, depicted by the blue curve, starts with a hyperbolic expansion at the nascent stages upon plasma separation event where the speed is at its highest value. Then, the speed of expansion decreases during the first $\sim 10 \mathrm{Gyr}$, due to gravity, until it reaches its minimal value at the phase transition at $\sim 10$ Gyr. Next, the Hubble parameter starts to increase in the reverse direction (the negative sign for the speed in the second phase indicates the opposite direction, as shown in Figure 3), which is the result of both sides starting to free-fall towards each other under gravitational acceleration. The Hubble parameter then reaches its zenith as it nears the Big Crunch.
According to the mechanics, the opposite signs of the acceleration (green curve) and the expansion speed in the first phase indicate that the expansion rate is slowing down, while the matching signs in the second phase indicate that the expansion speed is increasing, i.e., accelerated expansion. Additionally, a rectified Hubble parameter, indicated by the orange curve, reflect expansion in the opposite direction during the second phase, so the integration constants are tuned to guide the mean evolution value of the parameter at $\sim 70 \mathrm{~km} \cdot \mathrm{s}^{-1} \mathrm{Mpc}^{-1}$.

\section{Universe Curvature}

\subsection{Simulation of Spacetime Continuum Curvature}

The spacetime worldlines of a single side are simulated based on the derived model in Eq. (16) along with nearby spacetime continuum worldlines corresponding to the early and present universe, as shown in Figure 5. In the early universe, simulations of spacetime worldline evolutions coupled with flat and positive initial curvatures are not equal at any age during the first phase, as shown in Figure 5a. This can reveal that the spacetime is curved similar to the curved surface of a ball, which aligns with early universe positive curvature observation according to PL18 (Di Valentino, Melchiorri and Silk, 2020).

Conversely, for the present accelerated phase of expansion in the reverse direction, the simulation of the evolution of spacetime worldlines coupled with positive initial curvature produce a flat end or flat spacetime, as shown in Figure 5b.

(a)

(b)
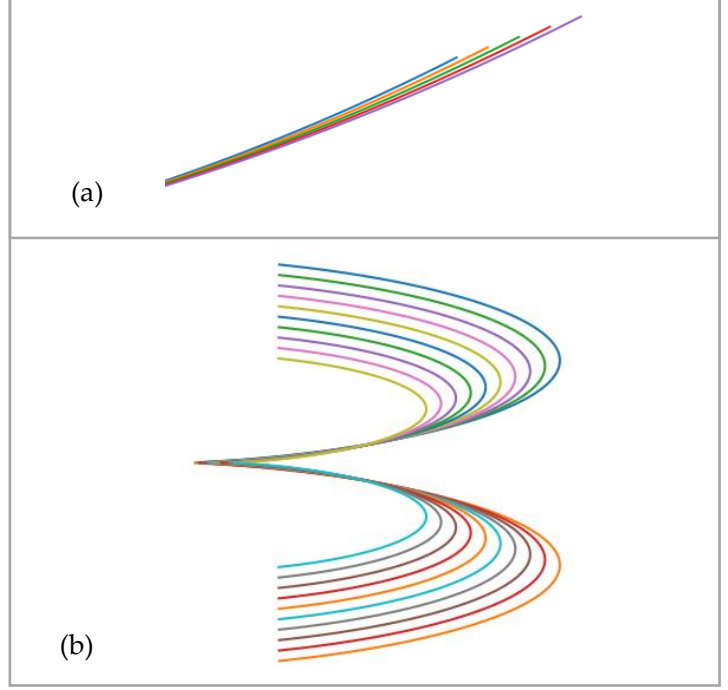

Figure 5. Evolution of spacetime worldlines corresponding to (a) the early and (b) the present universe. 
According to the derived model in Eq. (16) shown in Figure 3 and the simulated spacetime curvature in Figure 5, a schematic of a 2D spatial and 1D temporal dimensions is shown in Figure 6a, while radiation only worldlines according to Eq. (16) are predicted to pass from one side to another, which can explain the reason behind observing the $\mathrm{CMB}$ light while matter moves much slower than light. Figure $6 \mathrm{~b}$ shows an approximate apparent topology due to gravitational lensing effects.

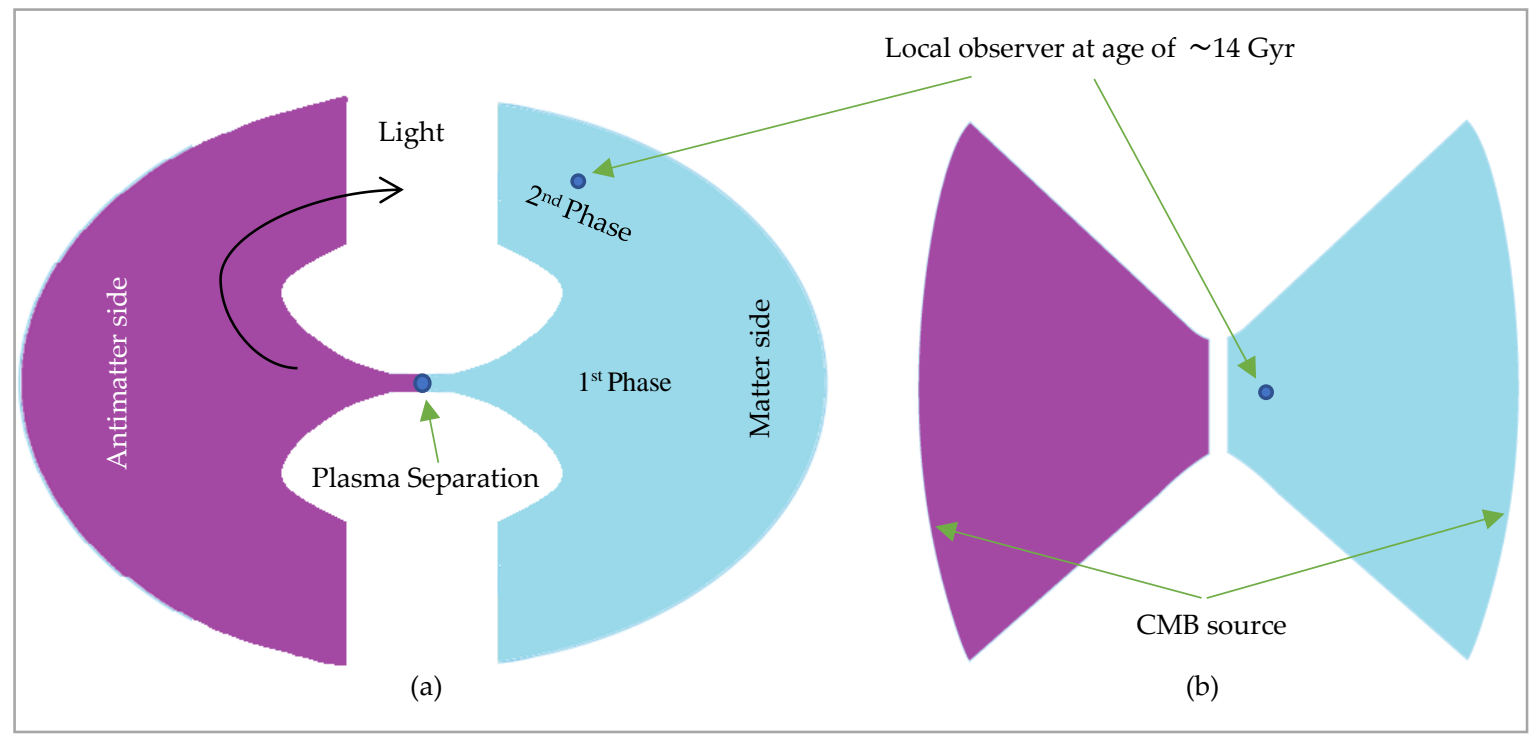

Figure 6. (a) 2D-schematic of the predicted cosmic topology of both sides at the first phase away from the early plasma while the second phase corresponding to the reversal of the expansion direction. (b) The apparent topology during the first and second phases caused by gravitational lensing effects.

Additionally, a 3D spatial and 1D temporal dimensions schematic of the spacetime evolutions of both sides of the universe is shown in Figure 7, where antimatter is shown to travel backwards in time as in

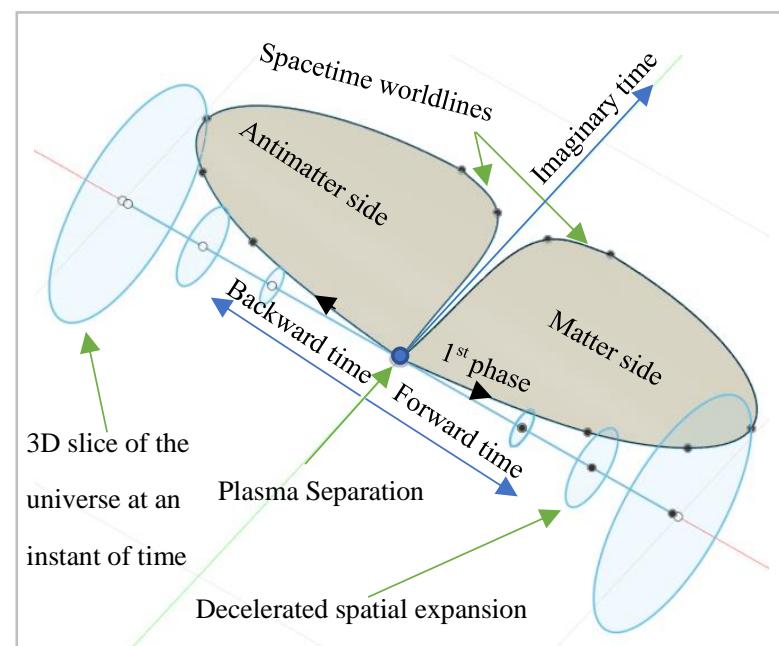

(a)
Feynman diagrams. The fine structure 'constant' is gradually evolving along the spacetime worldlines due to evolution of universe's density and remaining constant along with the perpendicular directions.

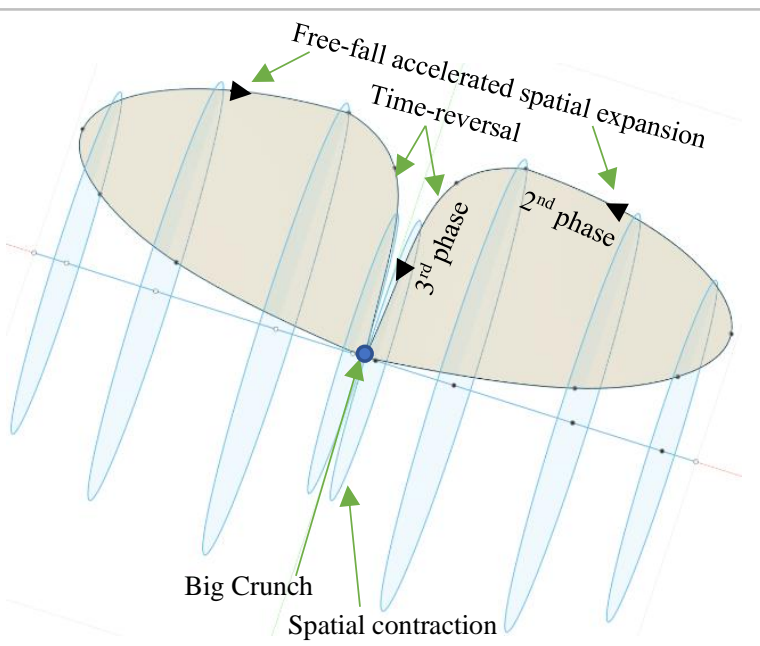

(b)

Figure 7. Schematic in 3D spatial and 1D temporal dimensions of both sides, according to the spacetime worldline evolution.

(a) In the first phase, both sides expand away from the early plasma. (b) In the second stage, both sides expand in reverse directions and free-fall towards each other at gravitational acceleration. In the third phase, both sides contract, due to high concentrations of matter/antimatter, leading to the Big Crunch. Blue circles represent a 3D universe slice, which is not necessarily a simply path connected. 


\subsection{Simulation of a Spiral Galaxy}

The consistent patterns of the galactic rotation curves observed using precise and independent galactic redshift data have confirmed that hydrogen clouds and outermost stars are orbiting galaxies at speeds faster than those calculated using Newtonian laws. Accordingly, the dark matter hypothesis was introduced to account for the apparently missing galactic mass and explain these fast-orbital velocities (Mannheim and Kazanas, 1989; Sofue and Rubin, 2000). However, no evidence for the existence of dark matter, which is hypothesised to account for the majority of galactic mass, has been observed since its introduction. The failure to find dark matter led to the introduction of new theories such as modified gravity and modified Newtonian dynamics (Chadwick, Hodgkinson and McDonald, 2013; Brouwer et al., 2016; Maeder, 2017; Van Meter, 2018; Milgrom, 2019). Several recent studies have reported that many galaxies do not contain dark matter (Guo et al., 2019). This scenario was used to inform galaxy formation simulations using modified Newtonian dynamics without considering the effects of dark matter (Wittenburg, Kroupa and Famaey, 2020). Therefore, it seems that there is no evidence for (or agreement on) the existence or nature of dark matter, and it may not be an essential requirement for galaxy formation.
Alternatively, because the shapes of spiral galaxies are highly similar to vortex shapes, a spiral galaxy can be modelled as a forced vortex, where external momentum is exerted on galaxies by the spacetime continuum curvature along its worldline evolutions. The derived model in Eq. (16) predicted that the curvature of spacetime continuum increases along its worldline evolution, with the highest degree of curvature occurring at the phase transition, as shown in Figures 3 and 6a. It can be inferred the fastorbital speeds observed for outer stars are a result of the variation of spacetime continuum curvature.

To evaluate this inference, a fluid simulation study was performed based on Newtonian dynamics using the Fluid Pressure and Flow software (Sam Reid et al, 2013). In this simulation, a perfect fluid of mass density $\rho$ and pressure $p$ was assumed to represent the spacetime continuum while the fluid particles were traced to study the divergence of fluid flux. Using these conditions, a fluid model was built to simulate and analyse the spacetime continuum flux for incrementally flatter worldline curvature, as shown in Figure 8a.

Using the resultant momentums in the fluid modelling and simulation study, a simulation of a spiral galaxy as a forced vortex is created, as shown in Figure 8b.

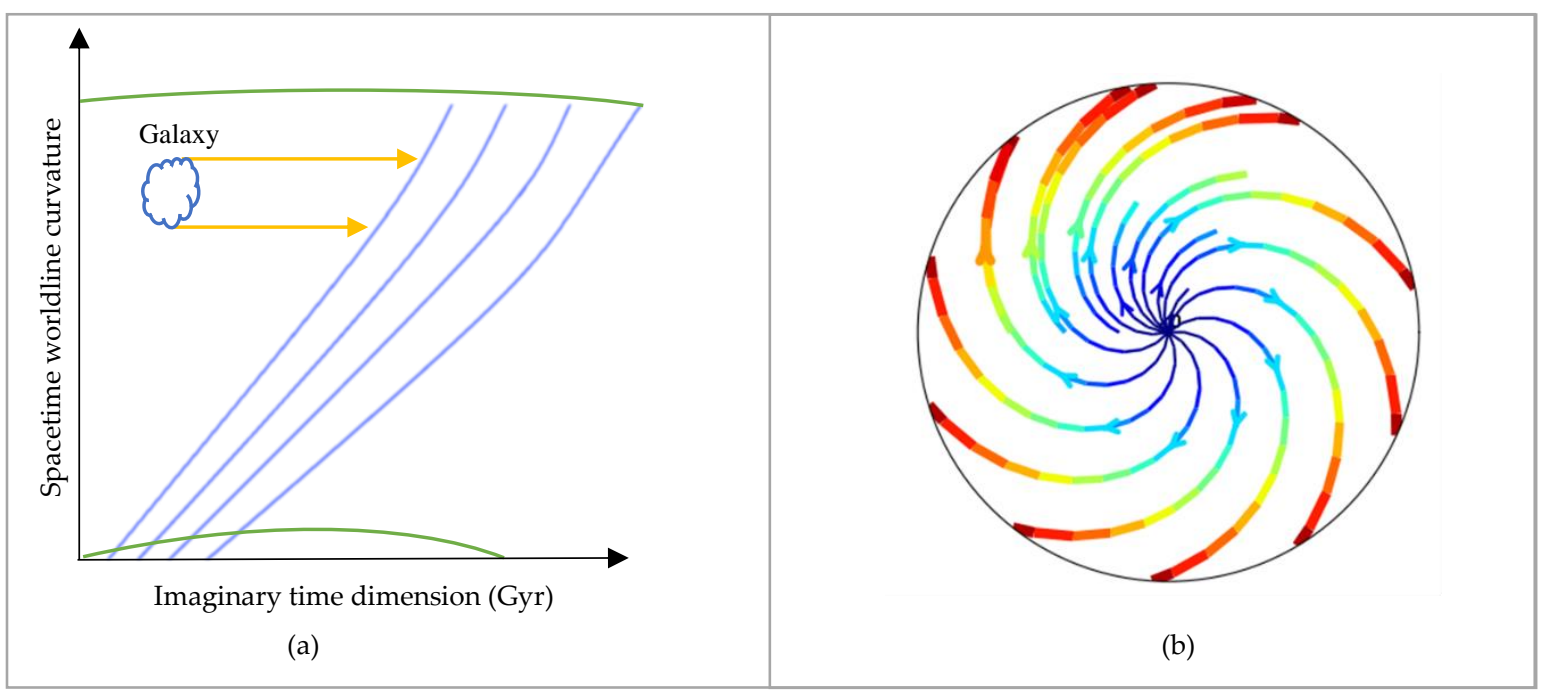

Figure 8. a) External momentum exerted on a galaxy because of the divergence of spatial curvature through the imaginary time dimension along the predicted spacetime worldlines. Green curves represent the curvature of spacetime worldlines. Blue curves represent the simulated spacetime continuum flux. b) Spiral galaxy rotation. Blue represents the slowest tangential speeds, and red represents the fastest speeds. 
The simulation shows that the tangential speeds of outer parts of the spiral galaxy are rotating faster in comparison with the rotational speeds of inner parts. Additionally, the galaxies of the same mass in the present universe were found to rotate faster than they were in the past because of the increase in the external momentum due to the highest spatial curvature at the phase transition. These results agree with the TullyFisher relation (Tully and Fisher, 1977). Based on the simulation results, it can be concluded the spacetime continuum curvature along its worldline evolution is responsible for the high speed of galaxies, explaining the effects attributed to dark matter.

\section{Conclusions}

In this study, the plasma of the early universe was modelled utilising enhanced FLRW metric. The evolution of the universe from early plasma was model utilising quantised spacetime continuum worldlines. The worldlines revealed two opposite solutions revealing that the universe has two sides: matter and antimatter.

The derived model predicted that a nascent hyperbolic expansion is followed by a phase of decelerating spatial expansion during the first $\sim 10$ Gyr, followed by a second phase of accelerating expansion; theoretically resolving the tension in Hubble parameter measurements. Both sides of the universe expand away from the early plasma during the first phase. Then, during the second phase, they reverse their directions and fall towards each other. It is conceivable that the matter and antimatter are freefalling towards, causing the current accelerating expansion of the universe. This can explain the effects attributed to dark energy. Further, the simulated curved spacetime during the decelerating phase was found to be flattened during the accelerating phase due to the reverse direction of the continuum worldlines; explaining the current space flatness.

Regarding the fast-orbital speed of stars, the simulation provided a physical explanation by which the spatial curvature through the imaginary time dimension along with the spacetime continuum worldlines of both sides was found to exert external momentum on galaxies. Thus, the geometrical spacetime curvature can be causing them to increase in speed, rather than the existence of dark matter.

Finally, the model predicted a final phase of time-reversal of spatial contraction leading to a Big Crunch, signifying a cyclic universe. The derived smallest possible reference radius of the early plasma due to its boundary gravitational contributions can reveal the early universe expansion upon emission of the $\mathrm{CMB}$ might mark the beginning of the universe from a previous collapse one.

\section{Future Work}

The integration constants of the derived model could be fine-tuned based on the astronomical data to accurately estimate the evolution of the universe. The accurate age could be calculated using the derived model in future works.

Funding: This research received no funding.

Acknowledgements: I am grateful to the Preprints Editor Ms Mila Marinkovic for her rapid and excellent attention in processing the submissions.

Conflicts of Interest: The author declares no conflict of interest

\section{Appendix A}

The Ricci curvature tensor $R_{u v}$ is solved using the Christoffel symbols of the second kind for the extended metric tensor $g_{\mu \nu}$ in Eq. (5), which are

$\Gamma_{\mu \nu}^{\rho}=\frac{1}{2} g^{\rho \lambda}\left(\partial_{\mu} g_{\lambda \nu}+\partial_{\nu} g_{\lambda \mu}-\partial_{\lambda} g_{\mu \nu}\right)$

Firstly, the solved non-zero Christoffel symbols are

\begin{tabular}{|c|c|}
\hline$\Gamma_{11}^{0}=\frac{a \dot{a}}{c^{2} a_{p}{ }^{2}\left(1-\frac{r^{2}}{r_{p}^{2}}\right)}$ & $\Gamma_{11}^{1}=\frac{r}{r_{p}^{2}\left(1-\frac{r^{2}}{r_{p}^{2}}\right)}$ \\
\hline$\Gamma_{22}^{0}=\frac{r^{2} a \dot{a}}{c^{2} a_{p}{ }^{2}}$ & $\Gamma_{22}^{1}=-r\left(1-\frac{r^{2}}{r_{p}^{2}}\right)$ \\
\hline$\Gamma_{33}^{0}=\frac{r^{2} a \dot{a} \sin ^{2} \theta}{c^{2} a_{p}^{2}}$ & $\Gamma_{33}^{1}=-r \sin ^{2} \theta\left(1-\frac{r^{2}}{r_{p}^{2}}\right)$ \\
\hline \multicolumn{2}{|c|}{$\Gamma_{01}^{1}=\Gamma_{02}^{2}=\Gamma_{03}^{3}=\Gamma_{10}^{1}=\Gamma_{20}^{2}=\Gamma_{30}^{3}=\frac{\dot{a}}{a}$} \\
\hline \multicolumn{2}{|c|}{$\Gamma_{12}^{2}=\Gamma_{21}^{2}=\Gamma_{13}^{3}=\Gamma_{31}^{3}=\frac{1}{r}$} \\
\hline$\Gamma_{33}^{2}=-\sin \theta \cos \theta$ & $\Gamma_{23}^{3}=\Gamma_{32}^{3}=\cot \theta$ \\
\hline
\end{tabular}


The Ricci curvature tensor is given by

$R_{\mu \nu}=\partial_{\lambda} \Gamma_{\mu \nu}^{\lambda}-\partial_{v} \Gamma_{\mu \lambda}^{\lambda}+\Gamma_{\mu \nu}^{\rho} \Gamma_{\rho \lambda}^{\lambda}-\Gamma_{\mu \lambda}^{\rho} \Gamma_{\rho \nu}^{\lambda}$

Secondly, the non-zero components of the Ricci tensor are solved as follows

The $t-t$ component is

$R_{t t}=R_{00}=-\partial_{0} \Gamma_{01}^{1}-\partial_{0} \Gamma^{2}{ }_{02}-\partial_{0} \Gamma_{03}^{3}-\Gamma^{1}{ }_{01} \Gamma^{1}{ }_{10}$ $-\Gamma_{02}^{2} \Gamma_{20}^{2}-\Gamma_{03}^{3} \Gamma^{3}{ }_{30}$

$R_{t t}=-3 \partial_{t} \frac{\dot{a}}{a}-3\left(\frac{\dot{a}}{a}\right)^{2}=-3 \frac{\ddot{a} a-\dot{a}^{2}}{a^{2}}-3 \frac{\dot{a}^{2}}{a^{2}}=-3 \frac{\ddot{a}}{a}$

The $r-r$ component is

$$
\begin{aligned}
& R_{r r}=R_{11}=\partial_{0} \Gamma_{11}^{0}-\partial_{1} \Gamma_{12}^{2}-\partial_{1} \Gamma^{3}{ }_{13}+\Gamma^{0}{ }_{11} \Gamma^{2}{ }_{02}+\Gamma^{0}{ }_{11} \Gamma^{3}{ }_{03} \\
& -\Gamma_{10}^{1} \Gamma_{11}^{0}+\Gamma_{11}^{1} \Gamma_{12}^{2}+\Gamma_{11}^{1} \Gamma_{13}^{3} \\
& R_{r r}=\partial_{t} \frac{a \dot{a}}{c^{2} a_{p}^{2}\left(1-\frac{r^{2}}{r_{p}^{2}}\right)}-2 \partial_{r} \frac{1}{r}+\frac{a \dot{a}}{c^{2} a_{p}^{2}\left(1-\frac{r^{2}}{r_{p}^{2}}\right)} \frac{\dot{a}}{a} \\
& +2 \frac{\mathrm{r}}{r_{p}^{2}\left(1-\frac{r^{2}}{r_{p}^{2}}\right)^{r}}-2 \frac{1}{r^{2}} \\
& R_{r r}=\frac{a \ddot{a}}{c^{2} a_{p}{ }^{2}\left(1-\frac{r^{2}}{r_{p}^{2}}\right)}+\frac{\dot{a}^{2}}{c^{2} a_{p}{ }^{2}\left(1-\frac{r^{2}}{r_{p}{ }^{2}}\right)}+\frac{\dot{a}^{2}}{c^{2} a_{p}{ }^{2}\left(1-\frac{r^{2}}{r_{p}^{2}}\right)} \\
& +\frac{2}{r_{p}^{2}\left(1-\frac{r^{2}}{r_{p}^{2}}\right)} \\
& R_{r r}=\frac{\left(\frac{a \ddot{a}}{a_{p}^{2}}+\frac{2 \dot{a}^{2}}{a_{p}^{2}}+\frac{2 c^{2}}{r_{p}^{2}}\right)}{c^{2}\left(1-\frac{r^{2}}{r_{p}^{2}}\right)}
\end{aligned}
$$

The $\theta-\theta$ component is

$$
\begin{aligned}
& R_{\theta \theta}=R_{22}=\partial_{0} \Gamma_{22}^{0}+\partial_{1} \Gamma_{22}^{1}-\partial_{2} \Gamma^{3}{ }_{23}+\Gamma_{22}^{0} \Gamma_{01}^{1}+\Gamma_{22}^{0} \Gamma^{3}{ }_{03} \\
& +\Gamma^{1}{ }_{22} \Gamma^{1}{ }_{11}+\Gamma^{1}{ }_{22} \Gamma^{3}{ }_{13}-\Gamma^{2}{ }_{20} \Gamma^{0}{ }_{22} \\
& -\Gamma_{21}^{2} \Gamma_{22}^{1}-\Gamma_{23}^{3} \Gamma_{32}^{3} \\
& R_{\theta \theta}=\partial_{t} \frac{r^{2} a \dot{a}}{c^{2} a_{p}{ }^{2}}-\partial_{r} \mathrm{r}\left(1-\frac{r^{2}}{r_{p}^{2}}\right)-\partial_{\theta} \cot (\theta)+\frac{r^{2} a \dot{a}}{c^{2} a_{p}^{2}} \frac{\dot{a}}{a} \\
& -\mathrm{r}\left(1-\frac{r^{2}}{r_{p}^{2}}\right) \frac{1}{r}-\cot ^{2}(\theta) \\
& R_{\theta \theta}=\frac{r^{2} a \ddot{a}}{c^{2} a_{p}{ }^{2}}+\frac{r^{2} \dot{a}^{2}}{c^{2} a_{p}{ }^{2}}+\left(3 \frac{r^{2}}{r_{p}{ }^{2}}-1\right)+\csc ^{2}(\theta)+\frac{r^{2} \dot{a}^{2}}{c^{2} a_{p}{ }^{2}} \\
& -\left(1-\frac{r^{2}}{r_{p}^{2}}\right)-\cot ^{2}(\theta) \\
& R_{\theta \theta}=\frac{r^{2} a \ddot{a}}{c^{2} a_{p}^{2}}+2 \frac{r^{2} \dot{a}^{2}}{c^{2} a_{p}^{2}}+\left(2 \frac{r^{2}}{r_{p}^{2}}\right)-1+\csc ^{2}(\theta)-\cot ^{2}(\theta) \\
& R_{\theta \theta}=\frac{r^{2}}{c^{2}}\left(\frac{a \ddot{a}}{a_{p}^{2}}+\frac{2 \dot{a}^{2}}{a_{p}^{2}}+\frac{2 c^{2}}{r_{p}^{2}}\right)
\end{aligned}
$$

The $\phi-\phi$ component is

$$
\begin{aligned}
& R_{\phi \phi}=R_{33}=\partial_{0} \Gamma_{33}^{0}+\partial_{1} \Gamma_{33}^{1}+\partial_{2} \Gamma^{2}{ }_{33}+\Gamma^{0}{ }_{33} \Gamma^{1}{ }_{01}+\Gamma^{0}{ }_{33} \Gamma^{2}{ }_{02} \\
& +\Gamma_{33}^{1} \Gamma_{11}^{1}+\Gamma_{33}^{1} \Gamma_{12}^{2}-\Gamma_{30}^{3} \Gamma_{33}^{0} \\
& -\Gamma_{31}^{3} \Gamma_{33}^{1}-\Gamma_{32}^{3} \Gamma_{33}^{2} \\
& R_{\phi \phi}=R_{33}=\partial_{t} \frac{r^{2} a \dot{a} \sin ^{2} \theta}{c^{2} a_{p}{ }^{2}}-\partial_{r} \mathrm{r} \sin ^{2} \theta\left(1-\frac{r^{2}}{r_{p}^{2}}\right) \\
& -\partial_{\theta} \sin \theta \cos \theta+2 \frac{r^{2} a \dot{a} \sin ^{2} \theta}{c^{2} a_{p}{ }^{2}} \frac{\dot{a}}{a} \\
& -r \sin ^{2} \theta\left(1-\frac{r^{2}}{r_{p}^{2}}\right) \frac{r}{r_{p}^{2}\left(1-\frac{r^{2}}{r_{p}^{2}}\right)} \\
& -r \sin ^{2} \theta\left(1-\frac{r^{2}}{r_{p}^{2}}\right) \frac{1}{r}-\frac{\dot{a} r^{2} a \dot{a} \sin ^{2} \theta}{c^{2} a_{p}^{2}} \\
& +\mathrm{r} \sin ^{2} \theta\left(1-\frac{r^{2}}{r_{p}^{2}}\right) \frac{1}{r}+\sin \theta \cos \theta \cot \theta \\
& R_{\phi \phi}=R_{33}=\frac{r^{2} a \ddot{a} \sin ^{2} \theta}{c^{2} a_{p}{ }^{2}}+\frac{r^{2} \dot{a}^{2} \sin ^{2} \theta}{c^{2} a_{p}{ }^{2}}-\sin ^{2} \theta\left(1+3 \frac{r^{2}}{r_{p}^{2}}\right) \\
& +\sin ^{2} \theta-\cos ^{2} \theta+\frac{r^{2} \dot{a}^{2} \sin ^{2} \theta}{c^{2} a_{p}^{2}} \\
& -\sin ^{2} \theta\left(\frac{r^{2}}{r_{p}^{2}}\right)+\cos ^{2} \theta \\
& R_{\phi \phi}=R_{33}=\frac{r^{2} a \ddot{a} \sin ^{2} \theta}{c^{2} a_{p}{ }^{2}}+2 \frac{r^{2} \dot{a}^{2} \sin ^{2} \theta}{c^{2} a_{p}{ }^{2}}+2 \sin ^{2} \theta \frac{r^{2}}{r_{p}{ }^{2}} \\
& R_{\phi \phi}=\frac{r^{2} \sin ^{2} \theta}{c^{2}}\left(\frac{a \ddot{a}}{a_{p}{ }^{2}}+\frac{2 \dot{a}^{2}}{a_{p}{ }^{2}}+\frac{2 c^{2}}{r_{p}{ }^{2}}\right)
\end{aligned}
$$

Thirdly, the inverse metric tensor $g^{u v}$ is

$$
g^{u v}=\left(\begin{array}{cccc}
\frac{1}{c^{2}} & 0 & 0 & 0 \\
0 & -\frac{\left(1-\frac{r^{2}}{r_{p}^{2}}\right)}{\left(\frac{a^{2}}{a_{p}^{2}}\right)} & 0 & 0 \\
0 & 0 & \frac{-1}{\left(\frac{a^{2}}{a_{p}^{2}}\right) r^{2}} & 0 \\
0 & 0 & 0 & \frac{-1}{\left(\frac{a^{2}}{a_{p}^{2}}\right) r^{2} \sin ^{2} \theta}
\end{array}\right)
$$

Finally, the Ricci scalar curvature $R=R_{u v} g^{u v}$ equals the Ricci curvature tensor time the inverse metric tensor as follows

$$
R=R_{u v} g^{u v}=\frac{-6}{c^{2}}\left(\frac{\ddot{a}}{a}+\frac{\dot{a}^{2}}{a^{2}}+\frac{c^{2} a_{p}^{2}}{a^{2} r_{p}^{2}}\right)
$$




\section{References}

Aghanim, N. et al. (2020) 'Planck 2018 results. V. CMB power spectra and likelihoods', Astronomy \& Astrophysics. EDP Sciences, 89. doi: 10.1051/00046361/201936386.

Ahmadi, M. et al. (2020) 'Investigation of the fine structure of antihydrogen', Nature. Nature Research, 578(7795), pp. 375-380. doi: 10.1038/s41586-020-2006-5.

Al-Fadhli, M. B. (2020a) 'Extended General Relativity for a Curved Universe', Preprints. Preprints. doi: 10.20944/preprints202010.0320.v3.

Al-Fadhli, M. B. (2020b) 'On Spacetime Duality and Bounce Cosmology of a Dual Universe', Preprints. Preprints. doi: 10.20944/preprints202005.0250.v7.

Albert Robson, B. (2019) 'Introductory Chapter: Standard Model of Cosmology', in Redefining Standard Model Cosmology. IntechOpen. doi: 10.5772/intechopen.85605.

Brouwer, M. M. et al. (2016) 'First test of Verlinde's theory of Emergent Gravity using Weak Gravitational Lensing measurements', Monthly Notices of the Royal Astronomical Society. Oxford University Press, 466(3), pp. 2547-2559. doi: 10.1093/mnras/stw3192.

Chadwick, E. A., Hodgkinson, T. F. and McDonald, G. S. (2013) 'Gravitational theoretical development supporting MOND', Physical Review D - Particles, Fields, Gravitation and Cosmology. American Physical Society, 88(2), p. 024036. doi: 10.1103/PhysRevD.88.024036.

Chiang, Y.-K. et al. (2020) 'The Cosmic Thermal History Probed by Sunyaev-Zeldovich Effect Tomography', arXiv. arXiv. doi: 10.3847/1538-4357/abb403.

Creswell, J. et al. (2017) 'On the time lags of the LIGO signals', Journal of Cosmology and Astroparticle Physics. Institute of Physics Publishing, 2017(8), p. 013. doi: 10.1088/1475-7516/2017/08/013.

Dirac, P. A. M. (1938) 'A new basis for cosmology', Proceedings of the Royal Society A: Mathematical, Physical and Engineering Sciences. The Royal Society, 165(921), pp. 199-208. doi: 10.1098/rspa.1938.0053.

Donoghue, J. F. (2003) 'Spatial and temporal gradients in the cosmological constant', Journal of High Energy Physics. Institute of Physics Publishing, 7(3), pp. 11151129. doi: 10.1088/1126-6708/2003/03/052.
Dyer, E. and Hinterbichler, K. (2009) 'Boundary terms, variational principles, and higher derivative modified gravity', Physical Review D - Particles, Fields, Gravitation and Cosmology, 79(2). doi: 10.1103/PhysRevD.79.024028.

Efstathiou, G. (2003) Is the low cosmic microwave background quadrupole a signature of spatial curvature?, Mon. Not. R. Astron. Soc. doi: 10.1046/j.13658711.2003.06940.x.

Ellis, G. F. R. and van Elst, H. (1998) 'Cosmological models (Cargl' $\{$ e $\}$ se lectures 1998)'. Available at: http://arxiv.org/abs/gr-qc/9812046.

Guo, Q. et al. (2019) 'Further evidence for a population of dark-matter-deficient dwarf galaxies', Nature Astronomy. Nature Research, 4(3), pp. 246-251. doi: 10.1038/s41550019-0930-9.

Holcomb, K. A. and Tajima, T. (1989) General-relativistic plasma physics in the early Universe, PARTICLES AND FIELDS THIRD SERIES.

Klein, O. (1966) 'Instead of cosmology', Nature. Nature Publishing Group, pp. 1337-1341. doi: $10.1038 / 2111337 \mathrm{a} 0$.

Klein, O. (1971) 'Arguments concerning relativity and cosmology', Science. American Association for the Advancement of Science, 171(3969), pp. 339-345. doi: 10.1126/science.171.3969.339.

Kozameh, C. et al. (1985) 'Conformal Einstein spaces', Springer.

Lachì Eze-Rey, M. and Luminet, J.-P. (2003) 'COSMIC TOPOLOGY', arXiv:gr-qc/9605010v2 9 Jan 2003.

Landau, L. D. (1986) Theory of Elasticity, Theory of Elasticity. Elsevier. doi: 10.1016/c2009-0-25521-8.

Lusso, E. et al. (2019) 'Tension with the flat \{ \Lambda\}CDM model from a high redshift Hubble Diagram of supernovae, quasars and gamma-ray bursts', Astronomy and Astrophysics. EDP Sciences, 628. doi: 10.1051/0004-6361/201936223.

Maeder, A. (2017) 'An alternative to the LCDM model: the case of scale invariance', The Astrophysical Journal. American Astronomical Society, 834(2), p. 194. doi: $10.3847 / 1538-4357 / 834 / 2 / 194$. 
Mannheim, P. D. and Kazanas, D. (1989) 'Exact vacuum solution to conformal Weyl gravity and galactic rotation curves', The Astrophysical Journal. IOP Publishing, 342, p. 635. doi: 10.1086/167623.

Meneghetti, M. et al. (2020) 'An excess of small-scale gravitational lenses observed in galaxy clusters', Science. American Association for the Advancement of Science, 369(6509), pp. 1347-1351. doi: 10.1126/science.aax5164. Van Meter, J. R. (2018) 'Dark-matter-like solutions to Einstein's unified field equations', Physical Review D. American Physical Society, 97(4), p. 044018. doi: 10.1103/PhysRevD.97.044018.

Migkas, K. et al. (2020) 'Probing cosmic isotropy with a new X-ray galaxy cluster sample through the L X- T scaling relation', Astronomy and Astrophysics. EDP Sciences, 636, p. 15. doi: 10.1051/0004-6361/201936602. Milgrom, M. (2019) 'MOND in galaxy groups: A superior sample', Physical Review D. American Physical Society, 99(4), p. 044041. doi: 10.1103/PhysRevD.99.044041.

Minami, Y. and Komatsu, E. (2020) 'New Extraction of the Cosmic Birefringence from the Planck 2018 Polarization Data', Physical Review Letters. American Physical Society, 125(22), p. 221301. doi: 10.1103/PhysRevLett.125.221301.

Pavel Grinfeld (2013) Introduction to Tensor Analysis and the Calculus of Moving Surfaces. Springer.

Penrose, R. (2005) The Road to Reality: A Complete Guide to the Laws of the Universe.

Riess, A. G. (2020) 'The expansion of the Universe is faster than expected', Nature Reviews Physics. Springer Nature, pp. 10-12. doi: 10.1038/s42254-019-0137-0.

Rugh, S. E. and Zinkernagel, H. (2000) 'The Quantum Vacuum and the Cosmological Constant Problem', Studies in History and Philosophy of Science Part B - Studies in History and Philosophy of Modern Physics. Elsevier Ltd, 33(4), pp. 663-705.

Ryden, B. (2006) Introduction to Cosmology. San Francisco, CA, USA: Addison Wesley, ISBN 0-80538912-1.

Ryskin, G. (2020) 'Vanishing vacuum energy', Astroparticle Physics. Elsevier B.V., 115, p. 102387. doi: 10.1016/j.astropartphys.2019.102387.

Sam Reid et al (2013) 'Fluid Pressure and Flow, PhET
Interactive Simulations.' University of Colorado. Sofue, Y. and Rubin, V. (2000) 'Rotation Curves of Spiral Galaxies', Annual Review of Astronomy and Astrophysics, 39(1), pp. 137-174. doi: 10.1146/annurev.astro.39.1.137. Straub, W. O. (2006) Simple Derivation of the Weyl Conformal Tensor, academia.edu. Available at: http://www.academia.edu/download/34935667/conformal .pdf.

Straumann, N. (2013) 'General Relativity (Graduate Texts in Physics)', in Springer. Springer.

Tully, R. B. and Fisher, J. R. (1977) 'A new method of determining distances to galaxies', Astron. Astrophys., 54, pp. 661-673.

Umetsu, K. (2020) 'Cluster-galaxy weak lensing', Astronomy and Astrophysics Review. Springer Science and Business Media Deutschland GmbH, pp. 1-106. doi: 10.1007/s00159-020-00129-w.

Uzan, J. P. (2011) 'Varying constants, gravitation and cosmology', Living Reviews in Relativity. Albert Einstein Institut, pp. 1-155. doi: 10.12942/lrr-2011-2.

Di Valentino, E., Melchiorri, A. and Silk, J. (2020) 'Planck evidence for a closed Universe and a possible crisis for cosmology', Nature Astronomy. Nature Research, 4(2), pp. 196-203. doi: 10.1038/s41550-019-0906-9.

Weinberg, S. (1972) 'Gravitation and Cosmology: Principles and Applications of the General Theory of Relativity'.

Wilczynska, M. R. et al. (2020) 'Four direct measurements of the fine-structure constant 13 billion years ago', Science Advances. American Association for the Advancement of Science, 6(17), p. eaay9672. doi: 10.1126/sciadv.aay9672. Wittenburg, N., Kroupa, P. and Famaey, B. (2020) 'The formation of exponential disk galaxies in MOND', The Astrophysical Journal. American Astronomical Society, 890(2), p. 173. doi: 10.3847/1538-4357/ab6d73. 